\title{
USE OF KNOWLEDGE INTENSIVE CAD (KIC) IN VIRTUAL PRODUCT VALIDATION
}

\author{
Daniel C.Y. Yip ${ }^{1}$, Henry M. C. Law ${ }^{1}$, Karen K. P. Cheng ${ }^{2}$, and Francis K.H. \\ $\mathrm{Lau}^{2}$ \\ ${ }^{l}$ G.E.W. Corporation Limited, 7/F, 2 Chong Yip Street, Kwun Tong, Kowloon, Hong Kong \\ ${ }^{2}$ Department of Industrial and Systems Engineering, The Hong Kong Polytechnic University \\ Tel. +852-2171-9800-Fax. +852-2345-8746 \\ E-mail:Daniel@gewcorp.com, Henry@gewcorp.com
}

\begin{abstract}
Use of $\mathrm{C} 3 \mathrm{P}(\mathrm{CAD} / \mathrm{CAE} / \mathrm{CAM} / \mathrm{PDM})$ in a product development cycle has been pursued as a strategic weapon and the most effective way to compete in the global marketplace. In reality, CAD alone is just a computer tool for 3D geometry creation whilst CAE still slow in its steps to solve a non-linear and multi-discipline design problem. In order to distinguish a manufacturing company from its peers, a KIC model which combines the use of the AI/neural network and CAE prediction for solving a non-linear and multi-discipline design problem is proposed. The model illustrates how experimental/legacy data can be extracted, crystallized and deployed to appraise the design of a dedicated product feature in a much simple manner. The methodology for the development of such a KIC system, its implementation and application will be thoroughly discussed and demonstrated through a case study concerning the design of an electrical toaster case.
\end{abstract}

Key words: Knowledge Design, Neural Network, Virtual Product Validation

\section{INTRODUCTION}

Due to the pressure induced by the global competition and the change of product development mode, most manufacturing companies have to migrate from solely production/original equipment manufacturing (OEM) to manufacture with product development nature /original design manufacturing (ODM). Such business movement leads to the urgent need of a shift from experience dependent to a new paradigm of computer assisted 
design and development. Through the use of the C3P (CAD/CAM/CAE/PDM) technologies, a product design can be created and validated virtually. The steps of a traditional/sequential product design and development process that includes: (i) creation of a concept design, (ii) fabrication of a preliminary prototype, (iii) verification, (iv) modification and (iv) tool fabrication can now be compressed through use of concurrent engineering.

The use of CAE software to evaluate or appraise a design proposal has been regarded as the state-of-the-art and the most efficient and cost effective way to cut down both product development time and cost. The knowledge required to appraise a particular design study can rely on a CAE software package whilst the design optimization steps can follow methods such as Taguchi [Bendell 1989; Lau 1995]. Through the use of the "what if" scenario, a less competent designer/engineer is now able to manage a highly complex design task. Furthermore, through the integration of the Axiom Design, CAD and CAE technologies, a structured customized product design and development system that can guarantee the success of a dedicated product design can be built [K.C. Chan 2000]. Up to this moment, the development of the CAD technology and its application software has reached its maturity and further enhancement will solely concern with the ease of use and interfacing with other software.

The pace of CAE software development has been increased significantly, but no single CAE software package is capable to predict all kinds of product design tasks. It would take a long time to make CAE become a panacea for all kinds of product design validation. Up to this moment, most CAE packages are design to handle problems of a specific type. To predict a complex design problem that involves multi-disciplines, such as structure, motion and fluid flow together, several CAE packages have to be used and the individual predictions have to be combined to give the final estimation. Design validations conducted in such way will not only involve a huge capital investment of CAE software and expertise but also the accumulated error from individual CAE prediction will ruin the overall accuracy. As a matter of fact, before the full maturity of CAE technology, a knowledge intensive CAD (KIC) system is required to work with CAE to tackle a multidiscipline/non-linear design problem to achieve maximum design output.

\section{MODEL AND PLATFORM FOR THE DEVELOPMENT OF KIC}

The concept of KIC is relative recent in the development field of using artificial intelligent (AI) that provides the capability of capture and deploy 
knowledge within a manufacturing company. For a dedicated product, it was found that design using KIC could reduce as much as $75 \%$ of total product design time [William C.Regli 1999]. In the past sixty years, AI had undergone substantial ups and downs. The birth of AI was in the early 1940s and the central nervous system was regarded as the first work recognized in the AI field [Warren McCulloch and Walter Pitts, 1940]. Johnson Neumann [1951] introduced the first neural network computer while John McCarthy [1958] brought together researchers interested in the study of machine intelligence, artificial neural nets and automata theory to form the Dartmouth Conference Workshop that gave birth to coin artificial intelligence as a new science in 1956. AI was considered as valuable tool to support a decision making and regarded as great ideas with great expectation in the 1960s. However, AI was disillusioned and funding cutback in the early 1970s [Michael Negnevitsky 2002] and the pace of AI was slow down. Until the past two decades (1980s - 1990s), the development of preliminary binary model had brought AI to a more mature expert technology and could be applied in different areas.

The concept of KIC can be applied to any particular conformation of a design in respect to meet its performance. It makes use a process-driven approach to override the human dependent approach to appraise a design alternative in less time. A KIC system can be designed to handle a single or a collection of dedicated design tasks. The model and platform for the development of a KIC system are shown in Figure 1 and 2 respectively. 


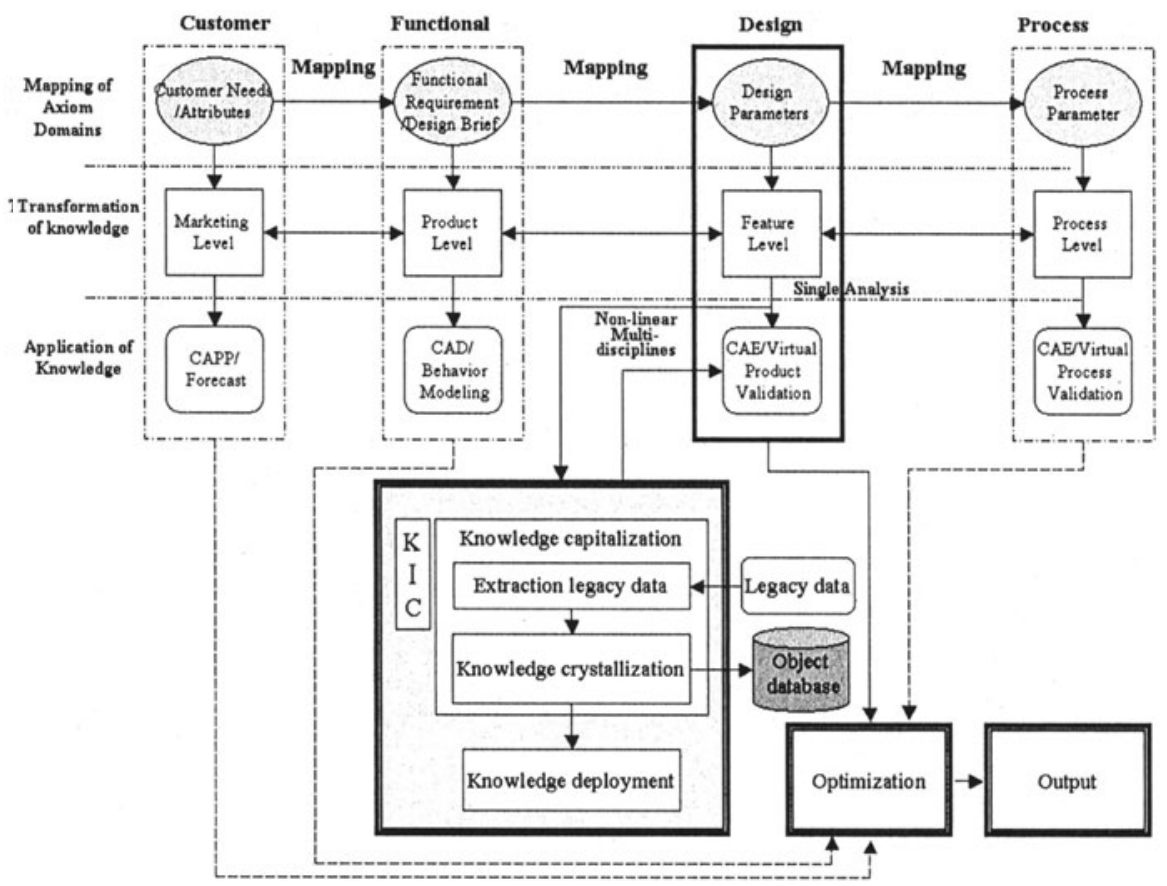

Figure 1. Model for the development of a KIC system

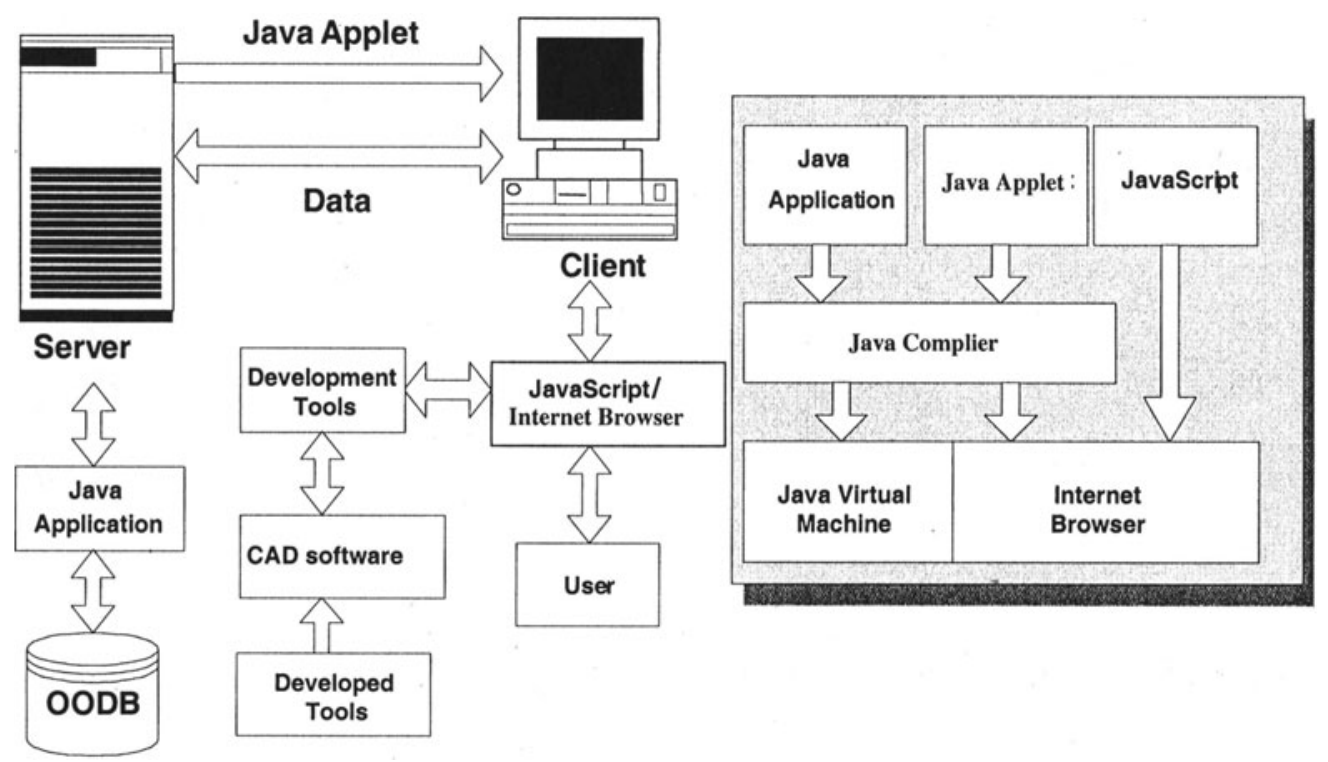

Figure 2. Platform for the development of a KIC system

The KIC system development process begins with the selection of a dedicated design task of a particular product. The development process for each individual knowledge module composes of two main phases: firstly, 
knowledge capitalization for the establishment of a knowledge database and secondly, the deployment of the codified knowledge database to form a system to handle and entertain any new inquiry. The architecture and main components of a KIC system are shown in Figure 3 and 4.

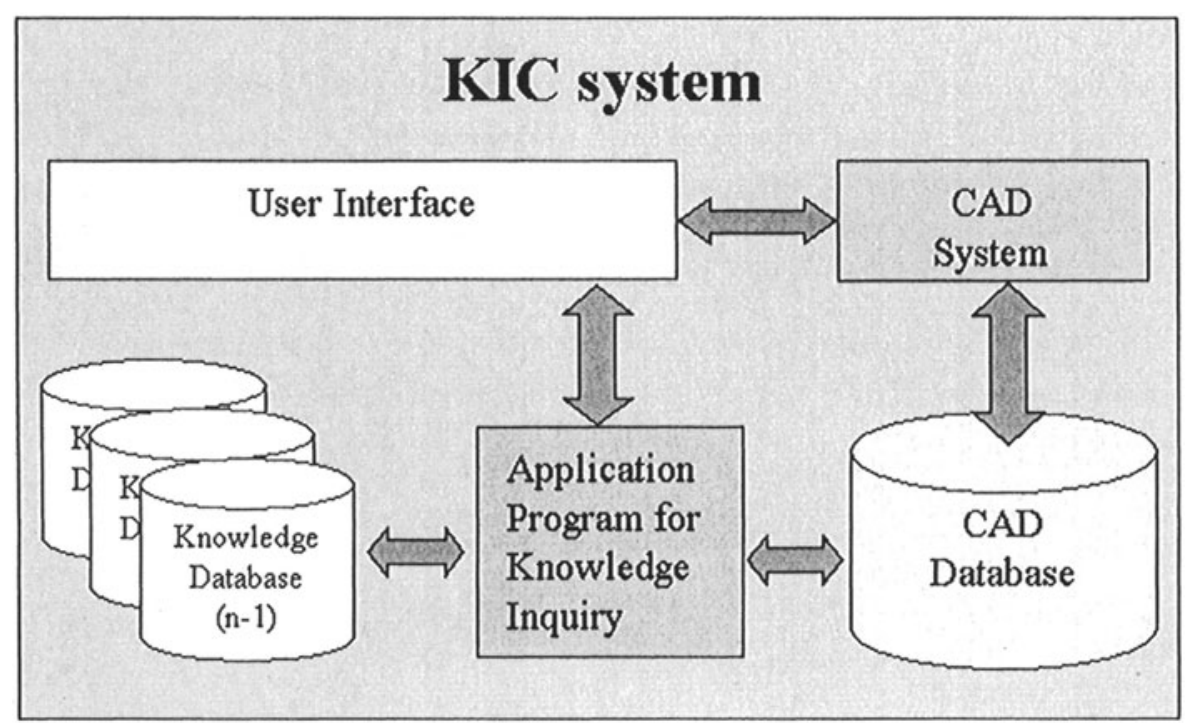

Figure 3. Architecture of the proposed KIC system

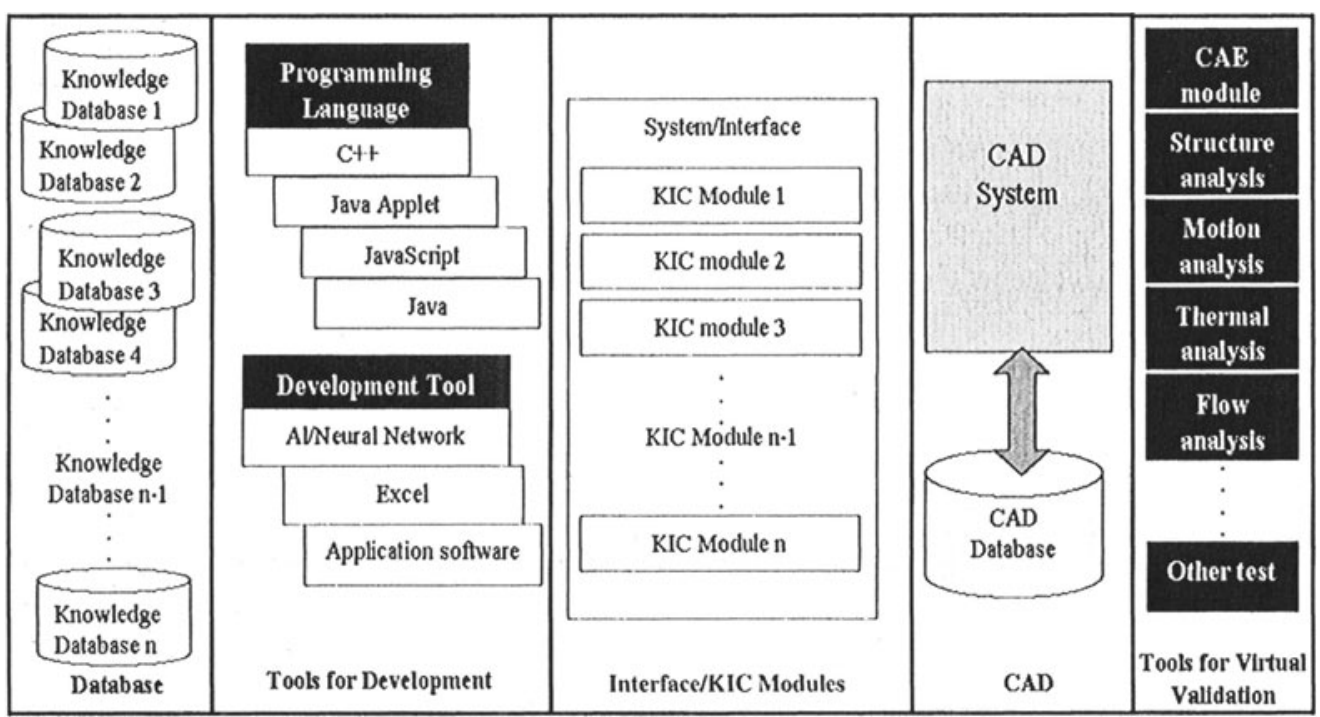

Figure 4. Components of a KIC platform 


\subsection{Knowledge Capitalization}

\subsubsection{Architecture For KIC Development}

The construction of an individual KIC module relies on a 3-tier client/server architecture that includes a client/interface, core/application, and back end/database (Figure 5). With the 3-tier architecture, modifications and new applications can be done off line and then assembly back without interfere the running of the KIC system whilst seamless upgrade is possible. A user will not notice change of system coding, as the interface or tools for a particular design task will remain the same. Depends on how a developer splits the application and the middleware that use to communicate between the tiers, many variations of multi-tier architectures are possible. The middle tier in 3-tier applications is not implemented as a monolithic program, instead, it can be used to collect components that use in a variety of design applications. Each application program can work for a dedicated design task. Such architecture can also distribute workloads across multiple servers and the tiers may reside on any computers. The 3-tier client/server architecture also permits a developer to start small both in scale and function and then expand the applications to intergalactic proportions. It permits the creation of individual application independently and then assembles system to create a new application and use Graphic User Interface (GUI) tools for access in web.

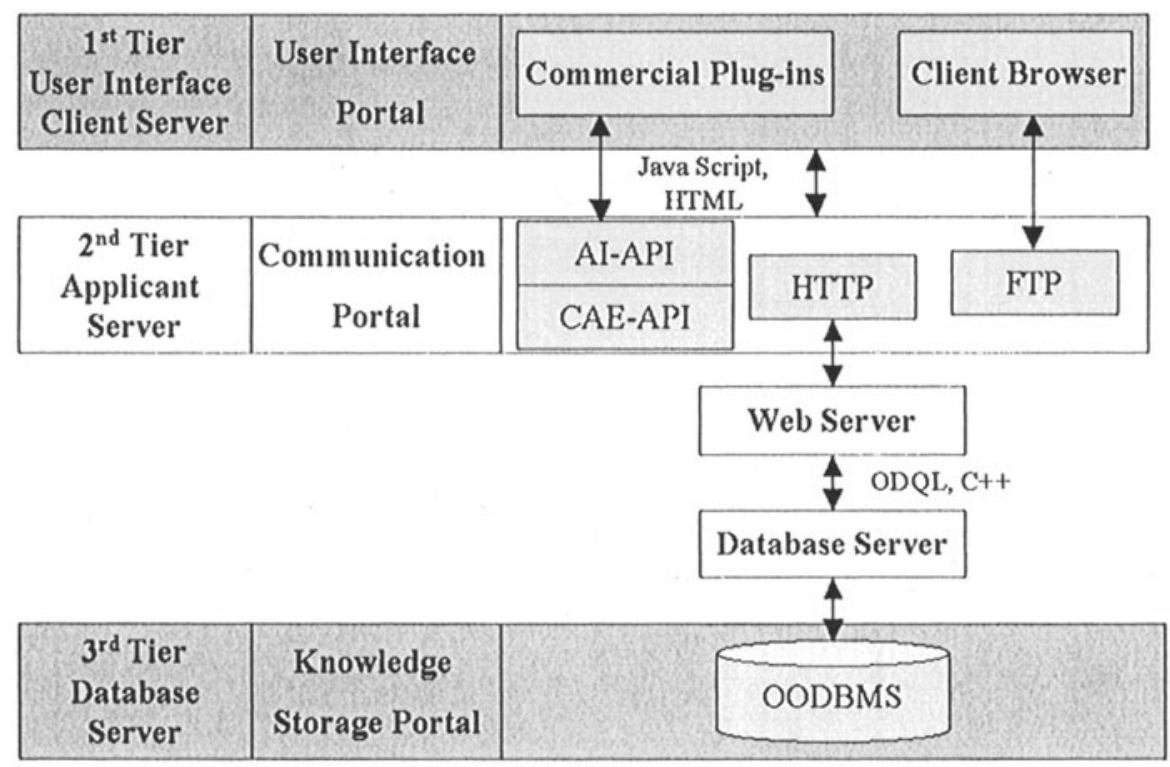

Figure 5. The 3-tier client/sever architecture 
The models for access through local, remote and Internet are shown in Figure 6. Through the use of a web browser, a user can login a KIC system any where with appropriate plug-in. The use of a Web Browser as an interface solves the problem of displaying heterogeneous data format in a single interface. With the plug-ins concept, nearly all the data output formats can be supported.

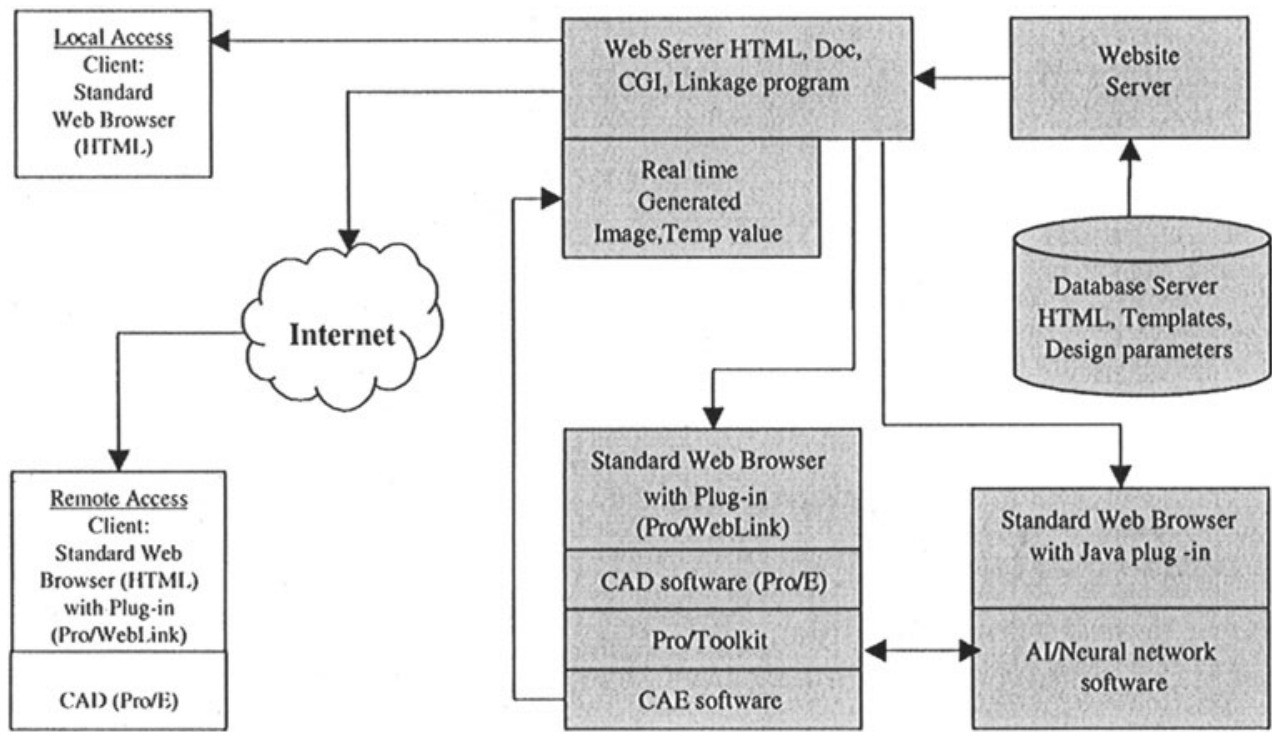

Figure 6. Models for local, remote and Internet accesses

\subsubsection{Data Extraction and Conversion}

In order to capitalize the required knowledge for a delicate design task, extraction and conversion of legacy data to the required format of a selected $\mathrm{AI} /$ neural network package is required. Legacy data can be classified into two types including: (i) structured (spreadsheets and relational databases) and (ii) unstructured (bitmap files and text documents). For textural data, extraction and conversion can be done through an automatic data handling (ADH) program (Figure 7). The data extraction process starts with reading in a template file for definition of searching criteria such as: subject name, coordinate attribute, start indicator and end indicator. Upon the completion o f a search, the found data fields will be stored in a CSV file and then export for data crystallization. The CSV file can also be converted into an ODQL file through the use of an ODQL converter and stored into an OODB for future processing.

For an unstructured data file, a complete data set can be imported to an OODB as an object property through the use of an ODQL programme. But if 
only some portion of a data set is required, a separate data extraction program has to be created (Figure 8) for the extracted of required data fields.

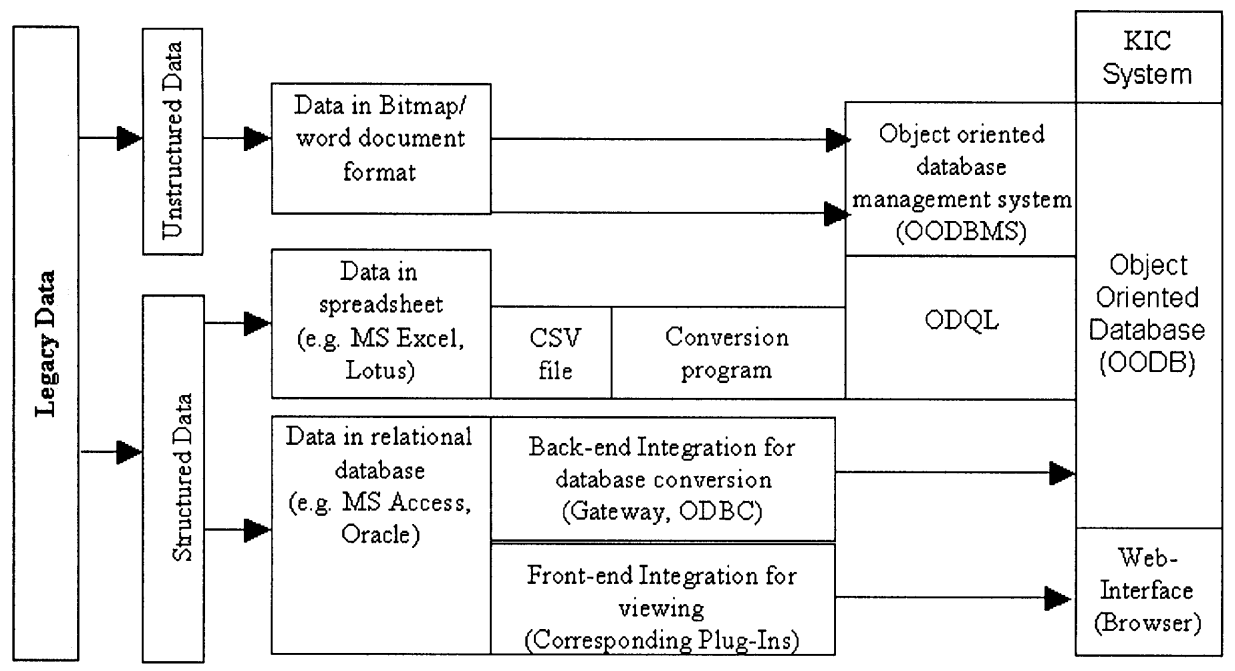

Figure 7. Automatic data handling system for legacy data extraction and conversion

Upon the completion of the legacy data collection, according to the nature of the design application and data properties, an appropriate neural network algorithm has to be selected for the crystallization of product knowledge. Figure 9 shows the model for the selection of a suitable AI/NN algorithm.

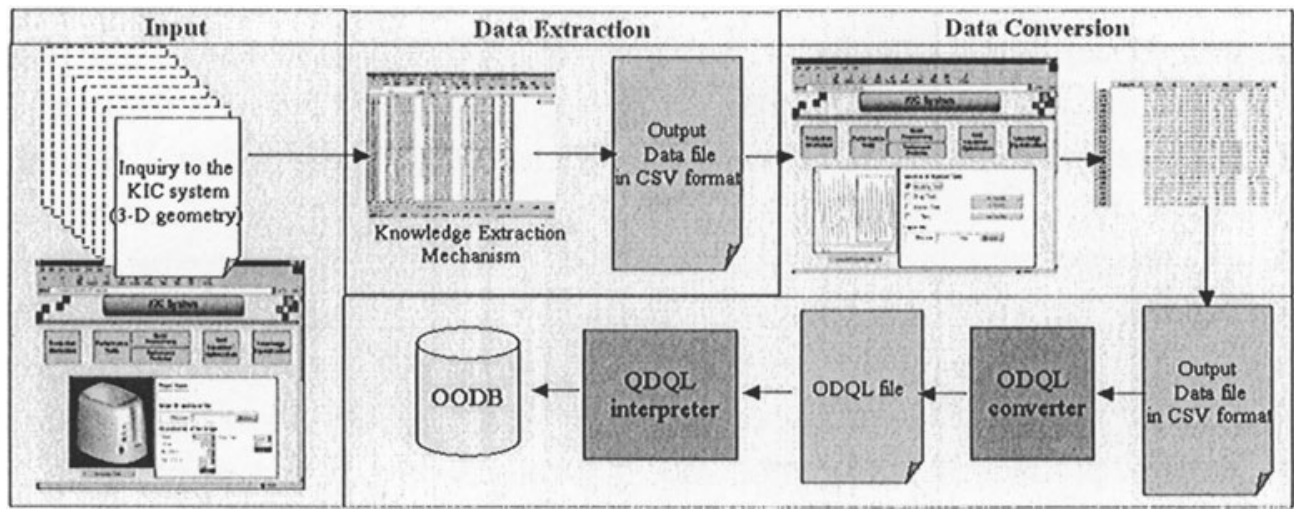

Figure 8. Workflow of extraction and conversion of legacy data 


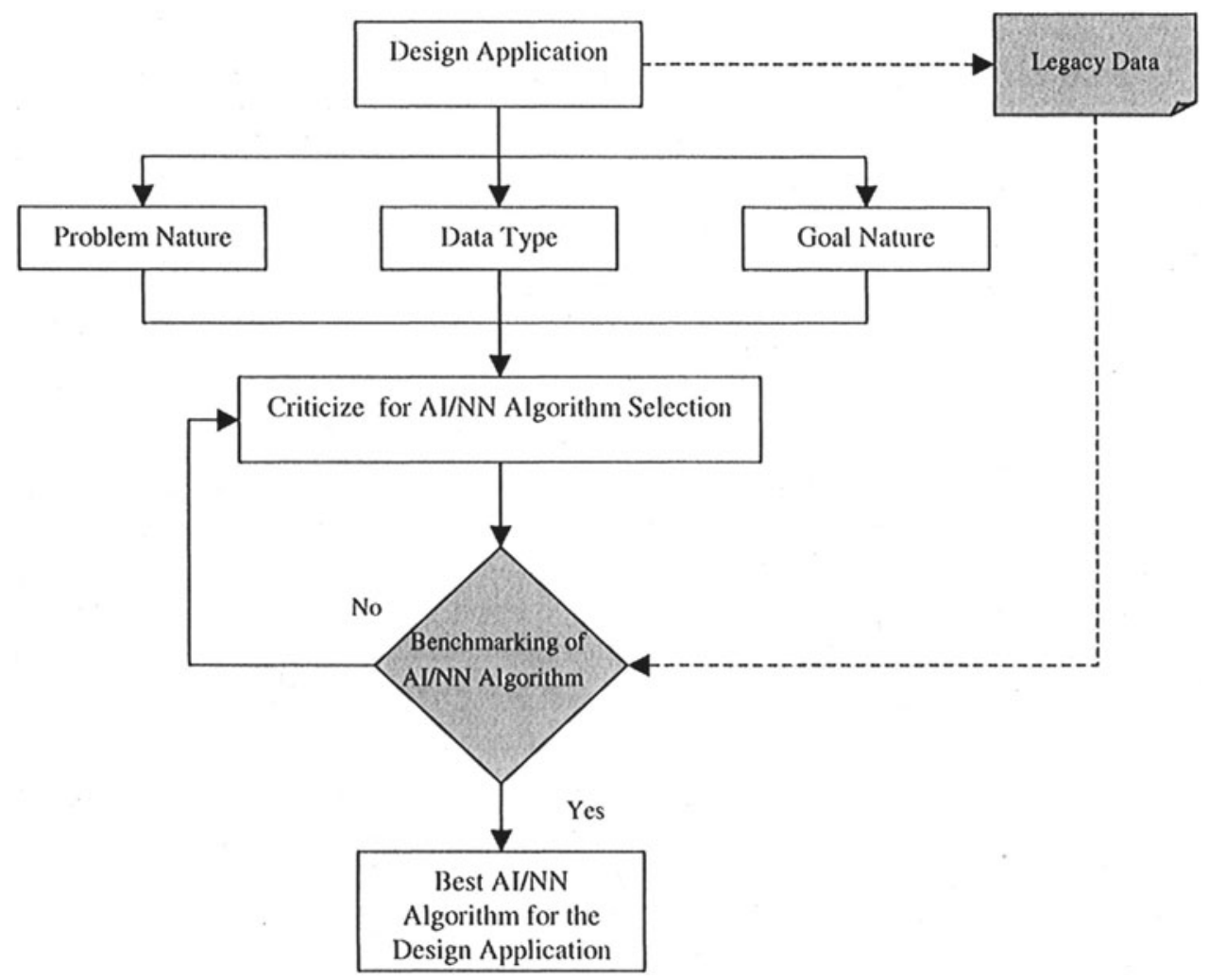

Figure 9. Model for the AI/NN algorithm selection

\subsubsection{Crystallization of Legacy Data}

Once an appropriate neural network algorithm has been found, a corresponding AI/NN software program of such nature can then be sought. Any noisy, erogenous, missing or irrelevant data sets are removed, purify and normalized whilst a schema for setup learning function and training has to be decided. Data sets are then trained to form neurons and their RMS error is checked. If the RMS error is within the target limits, then the performance of the neurons is accepted and further benchmarking/confirmation with the actual outcome can be done. If the RMS error indicates that the neurons are over fitted, then the number of data sets for generalization has to be reduced and re-training is required. In case the RMS error of the neurons' prediction fells below the targeted limits, additional/alternative training schema with adjustment of training parameters (e.g. epoch size and learning rate) is required for re-training. The final accepted neurons can then be output to form codified knowledge data base(s) and the crystallization process is then completed. The crystallization process might have to repeat several rounds that depend on the required 
quality of prediction. Figure 10 shows the workflow of the complete knowledge crystallization process.

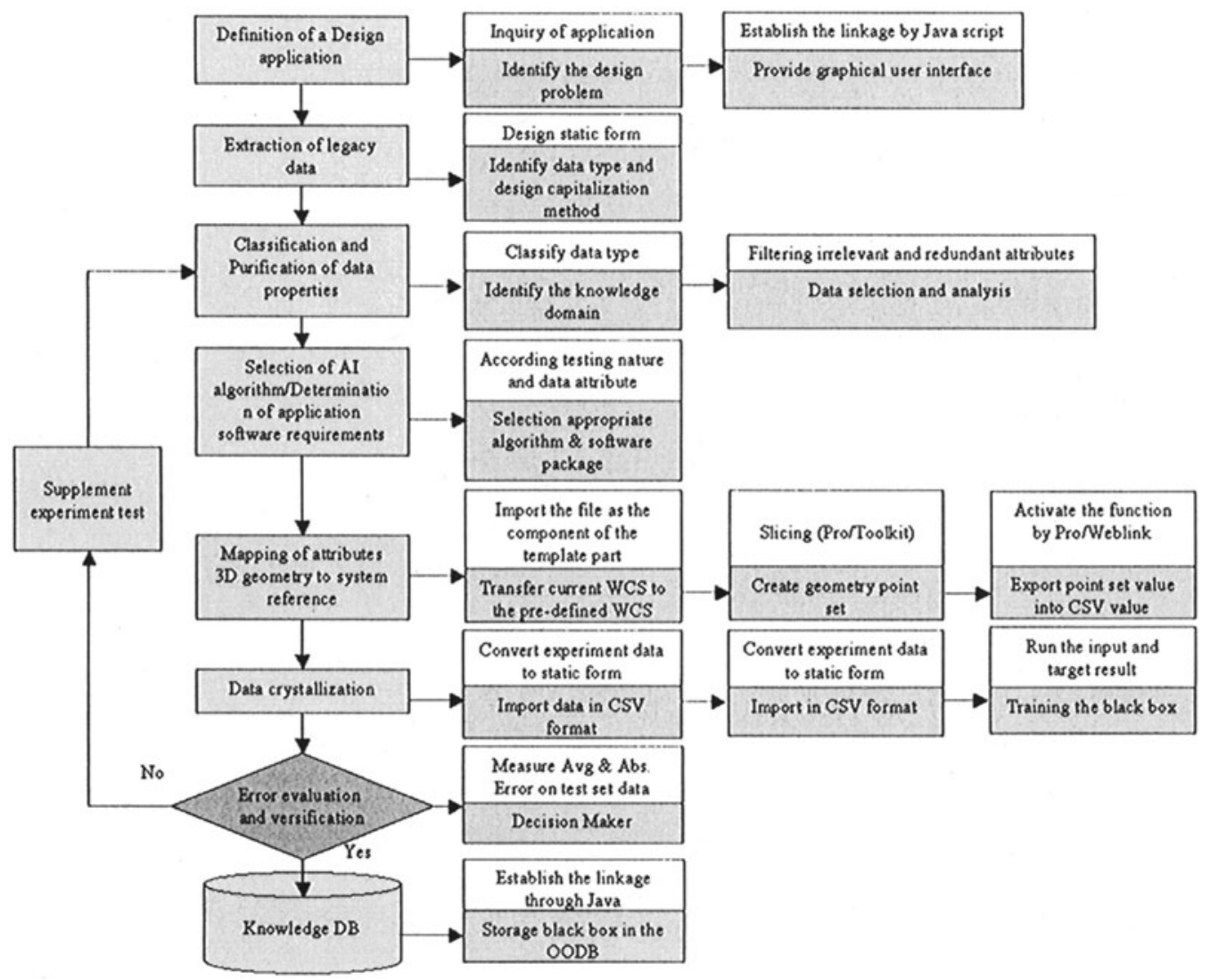

Figure 10. Workflow of the knowledge crystallization process

\subsection{Knowledge Deployment}

The second stage of a KIC development process is to construct the system for knowledge deployment. The development of the knowledge deployment system (Figure 11) composes of the creation of (i) a graphical user interface for the selection of a design application, input inquiry parameters, design attribute(s) and display of prediction(s)/output, (ii) an application program (API) for an inquiry interpretation - recognition of input attribute/feature recognition, design feature conversion and classification, (iii) an application program for knowledge generation/prediction and knowledge retrieval; and (iv) an application program for the conversion of textual information into a graphical display output. For example, to begin knowledge acquisition (heating test), the input attributes ( $\mathrm{x}, \mathrm{y}$ and $\mathrm{z}$ coordinates) is recognized through a slicing process to create inquiry coordinate(s) and convert into the system reference. Then the 
system will decide what knowledge should be extracted from the codified knowledge database to retrieve prediction(s) and then displayed in textual format. Upon the completion of the KIC deployment, a prototype KIC system should be built to appraise its performance. Any significant deviation of the prediction needs to be fed back and update the knowledge database.

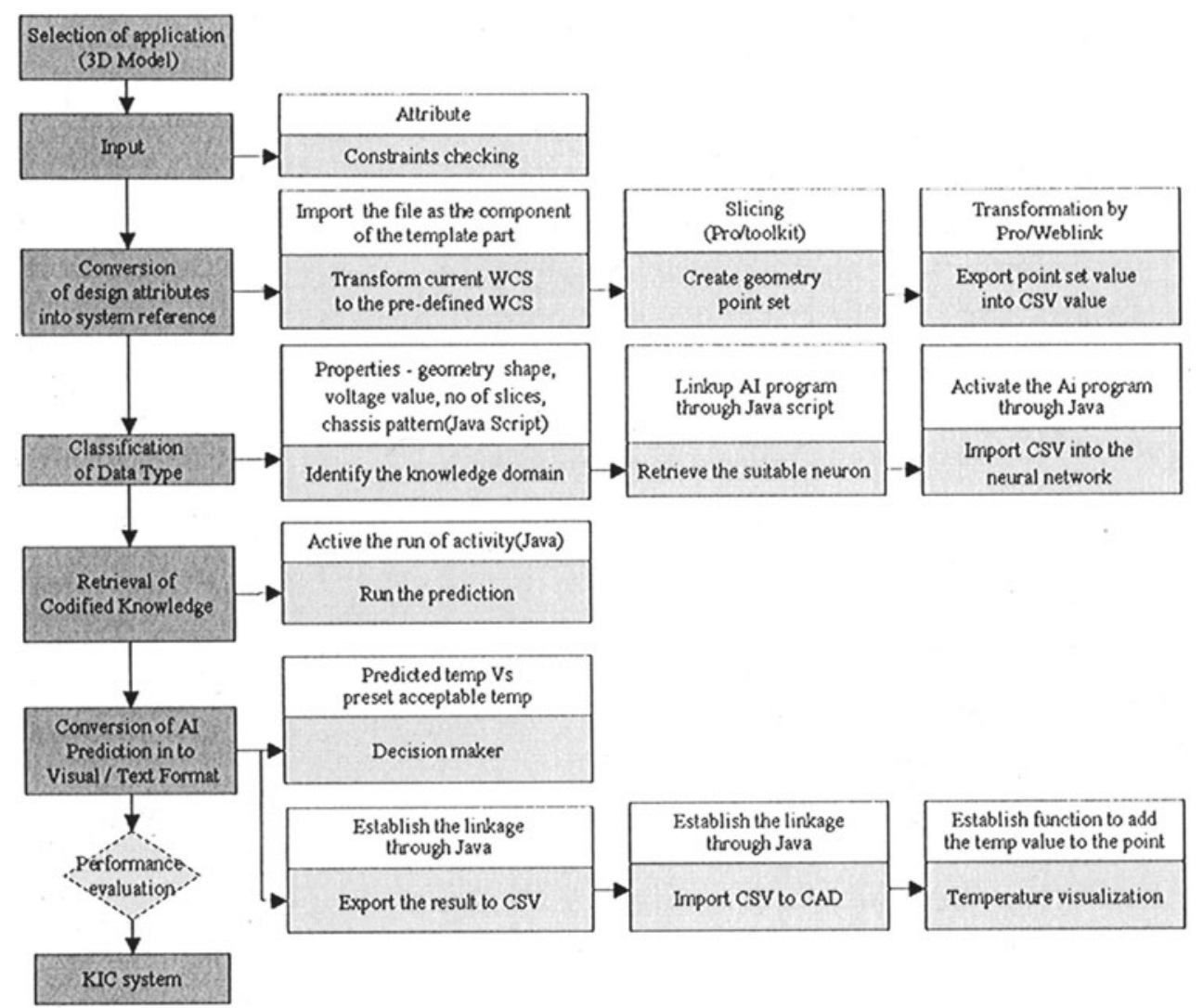

Figure 11. Workflow of the knowledge deployment process

\section{CASE STUDY - HEATING TEST OF A TOASTER CASE}

A KIC prototype with special concerns to meet the requirements of a heating test of a toaster case was built to illustrate the proposed model and system development methodology. The development of KIC system was conducted in one of the largest household electrical appliance manufacturing companies in Hong Kong and its execution had been divided into four phases:

1. Identification and prioritization of strategic product design improvement areas for KIC system development; 
2. Capitalization of the knowledge database and selection of the system development tools;

3. Deployment of the knowledge developed for a dedicated toaster case design application (heating test);

4. System integration, verification and benchmarking.

A model used for the study of the heating test according to IEC-CEI 335-3-9 is shown in Figures 12, 13, and 14. The heating test is aimed to confirm the maximum case temperature will not exceed $130^{\circ} \mathrm{C}$ for a particular case design. The variation of case temperature depends on (i) gap distance between the heating chassis and the case, (ii) airflow/area of openings in the bottom cover, (iii) profile of the case, (iv) case thickness, (v) plastic material and (vi) duration of heating. All these attributes will affect each other and the nature of the problem is non-linear and multidiscipline [Kristopher Seluga 1998]. In order to simulate such heating process virtually, several CAE software packages that can handle airflow, heat transfer (radiation, convection and conduction) and plastic stress/strain have to be use consecutively. Apart from the necessary of the CAE solvers and expertise to set the boundary conditions, the accuracy of the final virtual validation will be highly affected by the accumulated errors of individual software. Therefore, it is still not desirable or practical to solve a design problem of such kind by the multi-CAE packages approach. Legacy data of the past fifteen years including testing reports/modification requisition and other financial indicators such as quality cost were collected and transformed to the KIC system.

The KIC system development process followed the proposed model and workflows. Basic information theories, algorithm of the heating testing were classified and cataloged. The user-interface for the operation of the KIC system is shown in Figure 15. Its function includes (i) inquiry input, (ii) knowledge deployment, (iii) prediction, (iv) output, (v) legacy data extraction, (vi) conversion (vii) performance.

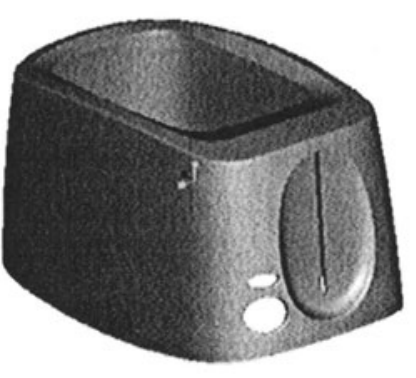

(a) Front Figure 12. Toaster case for the case study

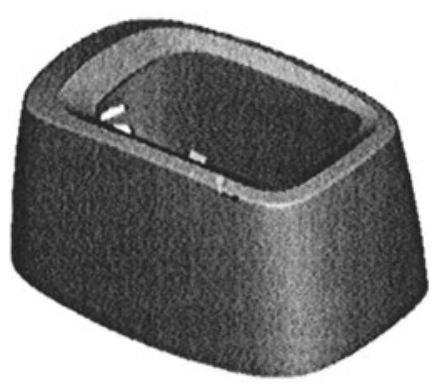

(b) Back 

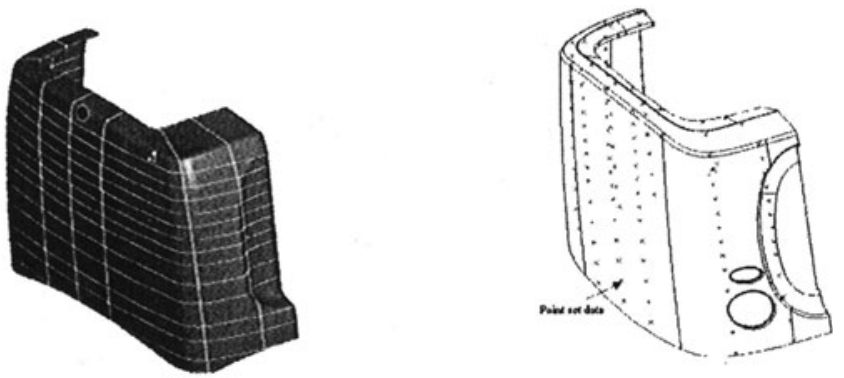

Figure 13. Screen shots for data recognition and mapping

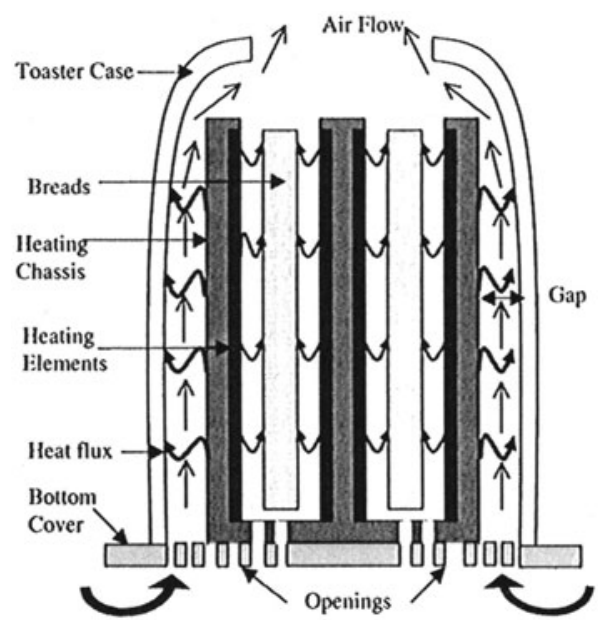

Figure 14. The heat transfer behavior of a toaster 


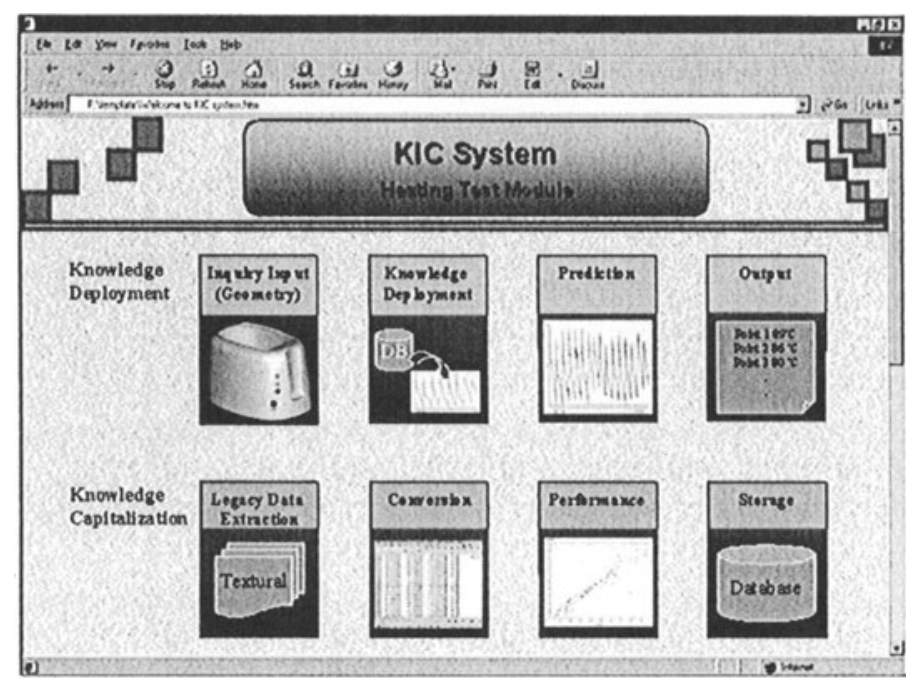

Figure 15. User interface of the KIC heating test module

Since the nature of the design task is modeling a function and the properties of the data are supervised, neural network algorithms including radial basis neural network (RBNN) and back-propagation (BP) can be used for the knowledge crystallization process. The development tools and software packages that use for the KIC integration was shown in Figure 16.

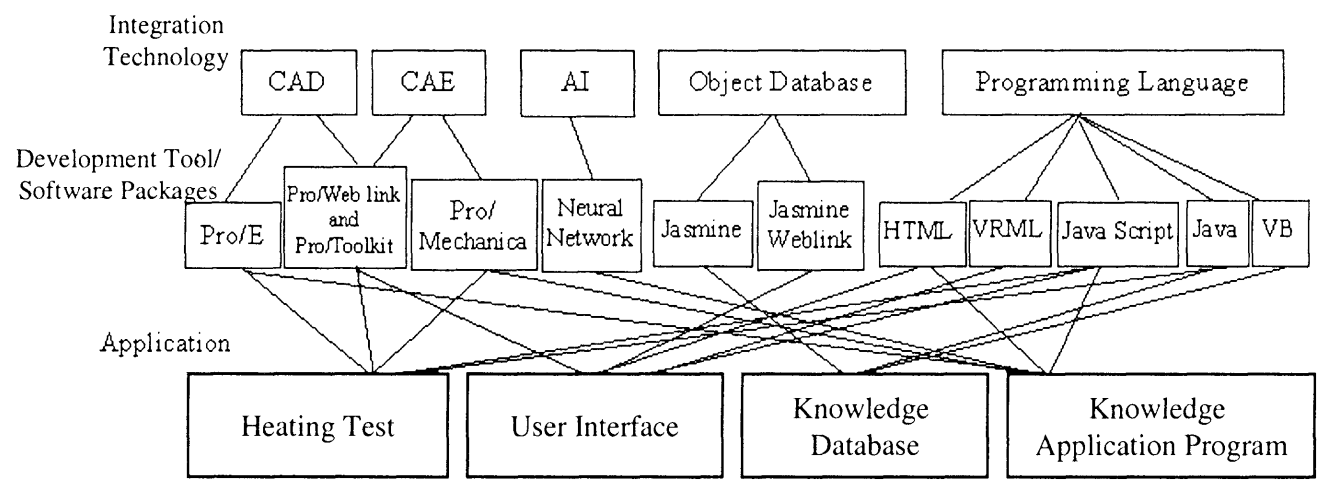

Figure 16. Development tools and software packages used for the KIC integration

Purified legacy data sets were trained by two commercial software packages to design knowledge. Upon the crystallization of the knowledge, a database CAD (Pro/Engineer) system was linked to display and tell the outcome of a design inquiry (Figure 17a). The prediction can be used as a boundary condition for subsequent thermal strain prediction (Figure 17b). 


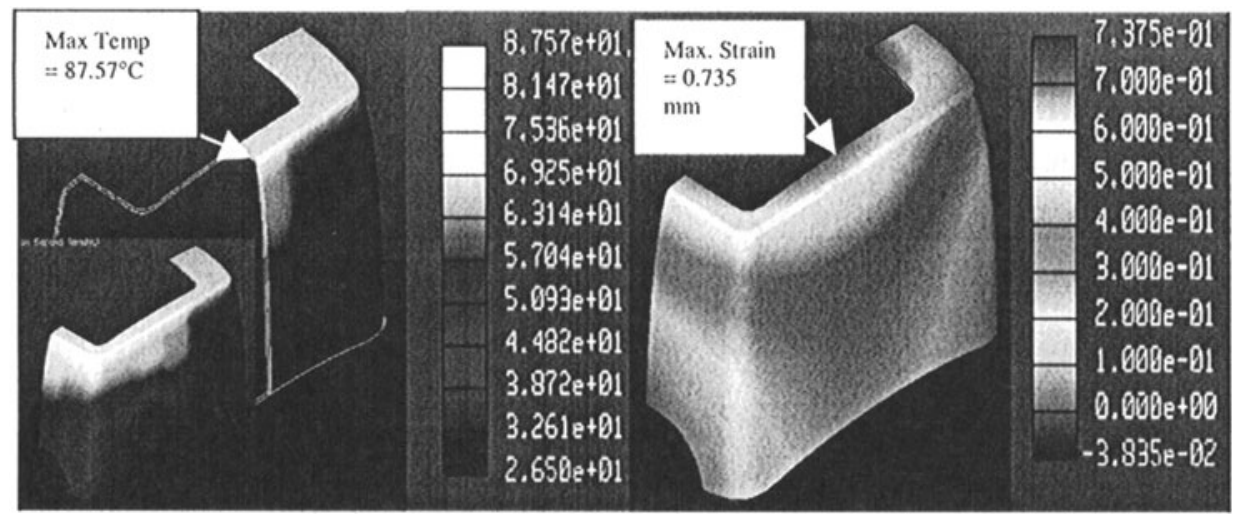

Figure 17. a) Temperature distribution under working condition, b) Prediction of thermal strain under working condition

\section{RESULTS AND DISCUSSION}

The accuracy of KIC prediction is highly dependent on the amount of legacy data input and the neural network algorithm selected for knowledge crystallization. The accuracy of RBNN versus BP for two different sets of data input (244 and 732 data sets) are summarized in table 1. whilst the comparison results of the predictions with actual findings are shown in Figure 18 and 19. It was found that the temperature predicted by RBNN is lightly accurate than BP. However, with regard to the mean and the maximum deviation of the temperature, RBNN is also better than BP. To concluded that, in this case, RBNN is far more suitable than BP.

From the virtual validation of thermal displacement, that the predicted temperature distribution as boundary condition, the predicted maximum strain was $0.735 \mathrm{~mm}$ (middle position of the toaster in the top), the deviation is only $5 \%$ (actual displacement was $0.7 \mathrm{~mm}$ ).

Table 1. Performance/accuracy of the neural network algorithms (RBNN and BP)

\begin{tabular}{llll}
\hline & Mean of temp & Max. Deviation & \% Error \\
\hline $\begin{array}{l}\text { Trial 1 (No of input } \\
\text { data set: 244) }\end{array}$ & & \\
RBNN & 8.85 & 22.35 & 15.84 \\
BP & 9.02 & 26.92 & 16.59 \\
Trail 2 (No of input & & & \\
data set: 732) & & & \\
RBNN & 5.04 & 20.74 & 9.16 \\
BP & 5.86 & 24.37 & 9.9 \\
\hline
\end{tabular}


In general, to solve a similar type of problem, it would cost over hundred thousands US dollars, but KIC and the engine just range from a few hundreds to several thousand.

Moreover, the electrical appliance manufacturing company claimed that it would need to take at least 3 weeks to do a real validation of this kind with an accuracy range from $70 \%$ to $80 \%$. With the proposed KIC approach, a complex non-linear multi-discipline design validation can be done in hours with acceptable accuracy.

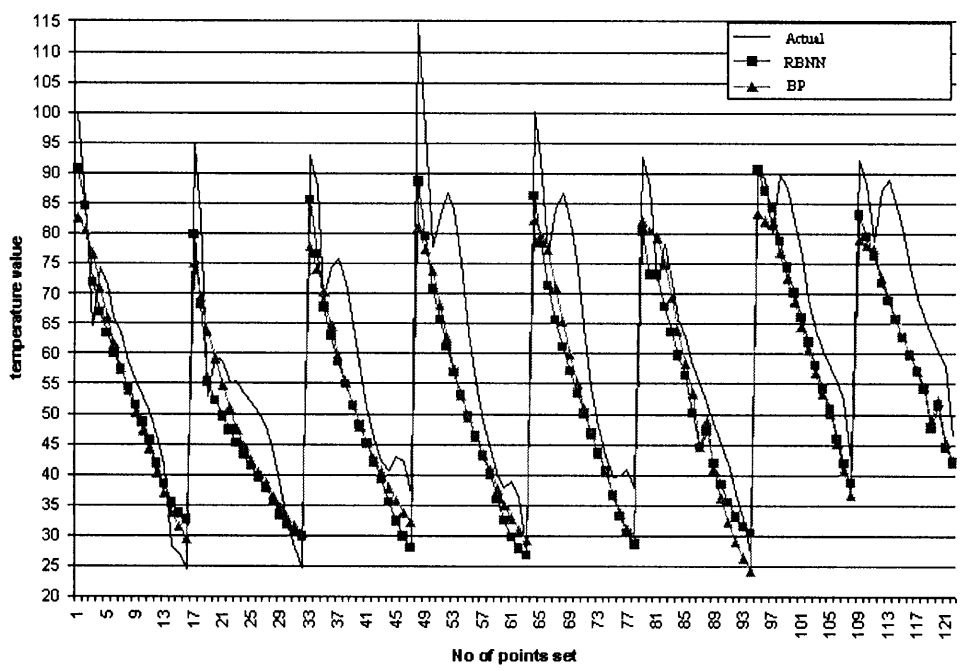

Figure 18. Comparison of RBNN and BP predictions with experimental results (244 point sets) 


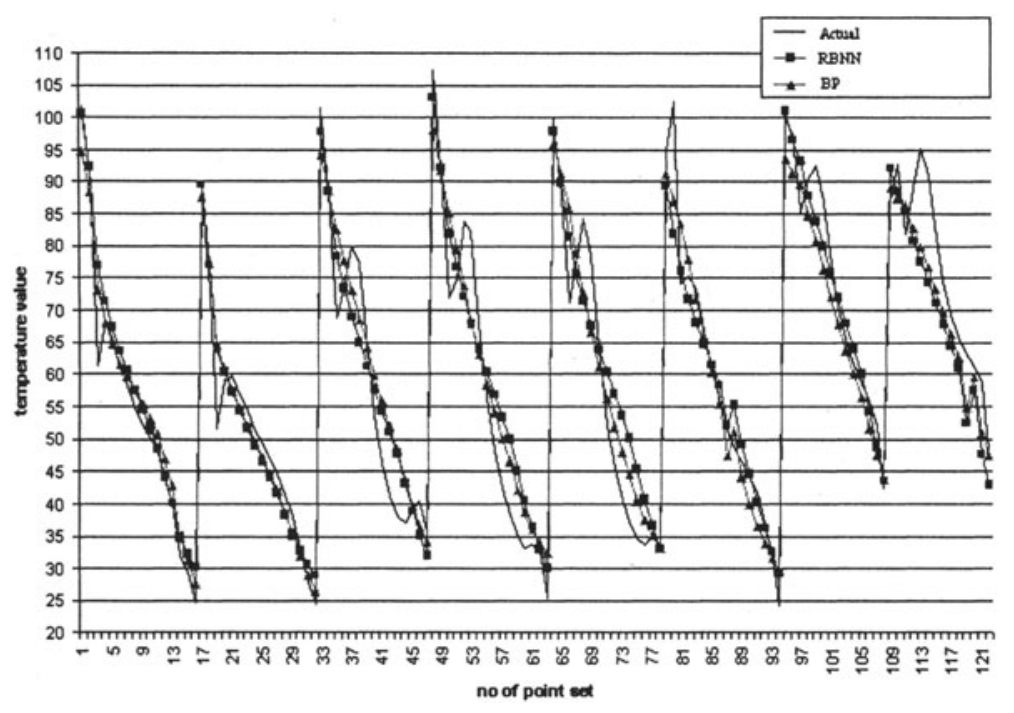

Figure 19. Comparison of RBNN and BP prediction with experimental results (732 point sets)

\section{CONCLUSION}

The model and workflow for the development of a KIC system to solve non-linear, multi-discipline design problem has been proposed and demonstrated. The KIC approach can provides a solution to avoid timeconsuming and costly multi-CAE approach. Legacy data can be reused through crystallization to form knowledge to aid rapid product development with less resource.

\section{ACKNOWLEDGEMENTS}

The work presented in this paper is supported by the Teaching Company Scheme (ZY22) of the Hong Kong Polytechnic University funded by the Industry Support Fund (UIT/26) and the G.E.W. Corporation Limited.

\section{REFERENCES}

Chan, K. W., Lau, K.H. and W.B. Lee. Development of a Web-based 3D system for Automatic Geometry Creation, 5th International Conference on Manufacturing Technology, Chiang Industrial Charity Foundation, 31 October to 3 November, CD ROM, 1999. 
Chan, K.C. and Lau, K.H. Market Oriented Digital Product Development Process, 8th International conference on Manufacturing Engineering, 2000, pp. 466-470.

He, Q. Neural Network and Its Application in IR, 1999, (http://alexia.lis.uiuc.edu/research/irg/uiuclis--1999-5+irg.pdf).

Johnvon, N. New directions in machine translation, Proceedings of the 12 the International Conference on computational Linguistics, Budapest, August 1988, pp. 18-19.

McCarthy, J. Programs with common sense, Proceedings of the Symposium on Mechanization of Thought processes, London, 1958, 1, pp. 74-84.

McCulloch, W.S. and Pitts, W. A logical calculus of the ideas immanent in nervous activity, Bulletin of Mathematical Biophysics, 1943.

Michael, N. Artificial Intelligence - A guide to intelligent system 2002, Addison-Wesley, 2002, pp. 5-18.

Seloga, K. Characterization of heat transfers interaction and efficiency of a household toaster, 1998, (http://www.lis.uiuc.edu/research/irg/uiuclis--1999-5irg.pdf).

William, C. and Regli, Digital Library support for engineering design and manufacturing, Proceedings of the 1999 ASME Design Engineering Technical Conferences, 1999, (http://gicl.mcs.drexel.edu/papers/PDFs/asme_cie_9080.pdf). 